\title{
The Application of Semantic Field Theory to English Vocabulary Learning
}

\author{
Chunming Gao \\ School of Foreign Languages, Changchun University of Science and Technology, China \\ Bin Xu \\ Kanrin Japanese School, Japan
}

\begin{abstract}
This paper explores the application of the semantic field theory in English vocabulary learning. It first investigates classifications of various semantic relations, including hyponym, antonymy, synonymy, etc. Then it illustrates with abundant examples how to employ each sense relation in learning vocabulary with the application of the semantic field theory. The study is of non-neglectable significance in that it helps to enlarge learners' vocabulary by constructing sense relations of new words and to help learners' mastery of vocabulary, so that they can learn English vocabulary more efficiently and systematically.
\end{abstract}

Index Terms —semantic field theory, hyponymy, antonymy, synonymy, English vocabulary learning

\section{INTRODUCTION}

\section{A. Significance of the Present Study}

Without doubt, English language plays a very important role in almost every field of modern world. It is a vital tool in communication. In the long history of English language, it has developed its individual uniqueness and has been widely used in most nations. Thus, it has become a true global language. At present, about 300 million people are learning English in China, and there are still more and more people joining the group of English learners. The number of English learners is rapidly increasing.

It is well known that vocabulary is of vital importance in language study because it is the essence of a language. Linguist D. A. Wilkins (1972) has the famous line "Without grammar very little can be conveyed, without vocabulary nothing can be conveyed. (p.111)" Truly, without vocabulary, there will be no sentence, no text and no language. Vocabulary is central to language and of crucial importance to the common language learner. The prominent role of vocabulary mastery in foreign language learning has been widely accepted and increasingly recognized. If we compare English to a giant architecture, then the bricks and concrete that made up the building will be English vocabulary. Obviously it is a common sense to us that the more concrete and bricks we use, the more stout and durable the architecture will be. Hence, if we have a great mastery of English vocabulary, we can surely win the first campaign of conquering English.

Basically, the history of English language can be divided into three periods: Old English, Middle English and Modern English. Through its development, English has gathered a considerable glossary which contains over a million words. However, most of these words are not widely used; many of them are even forgotten or solely banished. The English vocabulary that can be used in daily life is about twenty thousand, which to most English learners is still a large number to remember. In order to learn English more efficiently and gain a vocabulary that can meet the needs of normal communication, we should adopt an effective way to remember English words. Semantic field theory plays a surprisingly big part in learning English vocabulary effectively and systematically.

In recent years, more and more linguistic researchers start to pay close attention to the importance of semantic field theory for developing the language abilities and efficiency. Students find it difficult to remember all the English words at the same time because some words look very alike, such as considerate and considerable, temptation and temporary, geology and geometry, etc. Since semantic field theory studies the relationship between a series of words in a certain group, appropriate use of semantic field theory will make English vocabulary learning much easier.

\section{B. Theoretical Review of Semantic Field}

Before componential analysis emerged as a first concrete approach to describe word meaning, linguists distinguished and analyzed "semantic fields", that is, groups of words of which meanings are closely interrelated. Originally, "field" is a concept applied in physics which describes the layout condition of a certain object, such as electric field, magnetic field and gravitational field, which separately shows the layout principles of electricity, magnesium and gravity in a certain space. This concept was later applied into linguistics to describe the inter-relationship between different words. Gradually, it turned into a new linguistic approach called semantic field theory.

The study of semantic field theory is traced back to the very first idea of the founder of common linguistics, known as the famous German linguist from the middle of $19^{\text {th }}$ century, W. Humbodt. However, linguists who proposed the idea of 
semantic field theory on a real sense were some German and Swiss structuralism linguists from 1930s, such as Ipsen, Jolls, Porzig and J. Trier. Among all these brilliant linguists, J. Trier's idea is accepted most widely. People believe that his idea has brought semantics to a new level. Structuralist linguists abandoned the traditional research methods which are considered as isolated. Instead, a new research approach which studies semantics with relational and developmental viewpoints is widely accepted so as to focus on the unity of language system and the influence of contexts towards senses. (Zhou, 1997)

What is semantic field theory exactly? The core of semantic field theory is to analyze the relationship between genus and species of lexical study. (Mei, 1987) It suggests that the words of a language system are related with each other and they form a complete lexical system. In this system, certain words could form a semantic field under a common concept. For example, under the concept of stationery, pen, eraser, pencil, ruler and etc. could form a semantic field. In this semantic field, stationery represents the genus, or in other words, general concept; pen, eraser, pencil, and ruler represent species, or specific concept. In short, semantic field is a combination of a group of words that interact, dominate, distinguish and depend on with each other. The semantic range of the combination is called the field range of the semantic field.

Semantic field can be also called lexical field or domain, which refers to the combination of a bunch of words with interrelated meanings and dominated under a same concept. (Zhou, 2001) It has two folds of meanings. Some words, from a language, which were dominated under one certain concept, combined together and formed a semantic field. This certain concept may be represented by a superordinate, while the semantic field is formed from either hyponyms or semantic features. For example, under the common concept of furniture, words like table, closet, bed, bookshelf, couch, sofa, chairs, etc., could be gathered as a semantic field. Another example, burgundy, purple, pink, green, yellow, blue, orange, white and black, these words go to a common concept of color. On the other hand, words that belong to the same semantic field are not only semantically related, but also interact with each other. That is to say, when checking the meaning of a word, the first step is to compare the semantic meaning of the other words under the same semantic field. Take the antonymous semantic field formed by the word "fast" and slow/sober/loose/disloyal/faded/slowly/eat as an example, before determining the meaning of the word "fast", we will have to figure out the antonymous relations between fast and other words. For instance, fast means quick against slow, while it means fadeless against faded.

\section{Classifications of Semantic FIELD}

The semantic field theory was brought into its puberty by German scholar J. Trier in the 1930s, whose version is seen as a new phase in the history of semantics. Wu (1988, p.94-95) summarized Trier's semantic field theory as follows:

(1) The vocabulary in a language system is semantically related and builds up a complete lexical system. This system is unsteady and changing constantly. (2) Since the vocabulary of a language is semantically related, we are not supposed to study the semantic change of individual words in isolation, but to study vocabulary as an integrated system. (3) Since lexemes are interrelated in sense, we can only determine the connotation of a word by analyzing and comparing its semantic relationship with other words. A word is meaningful only in its own semantic field.

Trier's semantic field is generally considered paradigmatic. It deals with paradigmatic relations between words such as hyponymy, synonymy and antonymy. (Guo, p.51) This study follows this classification.

\section{A. Hyponymy}

Hyponymy is the most common branch of semantic field theory. It contains all objects that belong to the same category. The most fundamental category is dualistic, for example, paddy field and upland field, autorotation and revolution, etc. Another category is diversified, for example, navy, marine, air force, etc. Hyponymy can be multileveled, which means the basic level could be divided into more specific levels, and even more specific ones. For instance, we could divide animals into human being and birds and beasts, yet birds and beasts could be further divided into birds, fishes, insects and mammals, etc. And the mammals could also be specifically divided into pigs, dogs, cows, wolves and horses, etc. And finally, for example, horses can be further divided into broncos, stallions, ponies and so on. These divisions implicate the system of hyponymy, which is basically the superordinate-hyponym semantic field, also called general terms and specific terms. Take the example above for instance, if animals are the superordinate, human being, birds and beasts will be the hyponyms. On the other hand, if birds and beasts are taken as the superordinate, then birds, fishes, insects and mammals will be the hyponyms.

Hyponymy is featured as being relative. The relativity of this semantic field has two-fold meanings. On the one hand, it means that the superordinate-hyponym relations are not relative instead of absolute or unchanged. Take an example as the Graph 2-1-1 shows below:

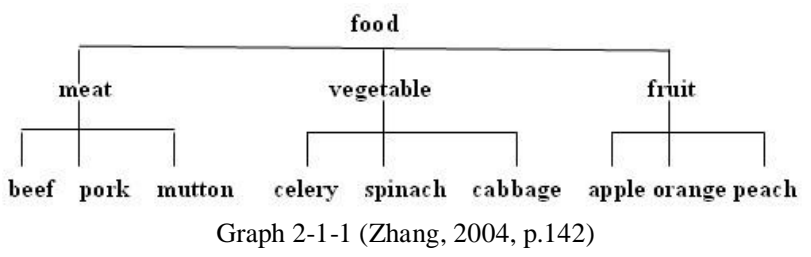


As the graph listed above, to food, meet is a hyponym and it locates at a lower level; while to beef, pork and mutton, meat is a superordinate and locates at a higher level. The same way works with vegetable, it is a superordinate towards celery, spinach and cabbage, yet it is a hyponym towards vegetable. On the other hand, when a semantic field was applied to describe different objects, the consulting standards are different and relative. For example, among the semantic field of big and small, the size is relative because sometimes the small ones are bigger than the big ones. For example, a small elephant is much bigger than a big mouse, and a small dog is bigger than a big butterfly.

\section{B. Antonymy}

Antonymy is another branch of semantic field which is formed by words with the same roots and range. Antonymy is usually formed with opposed, inconsistent and opposite meanings and therefore could be divided into three categories as follows:

(1) Polar Antonymy

Polar antonymy refers to the semantic field formed with the words of opposed and polar meanings. For example, hot-cold, rich-poor, etc. This type of semantic field has two features, semantic polarity and semantic relativity, which indicates that the semantic polarity only exists on the base of the semantic relativity. For example, we might say, $A$ man may be rich or very rich. One man may be richer than another. On the other hand, semantic relativity is gradual, which means we can insert words with different degrees between the two polars of the polar antonymy to show the hierarchy of the semantic relativity. For example, between hot and cold, we can insert warm, tepid, lukewarm and cool. Also, we can change the polar words from a polar semantic field to other words with different degrees to show the hierarchy of the semantic relativity. Take the same example; we could change hot or cold to boiling, sweltering, chilly or freezing. (Zhou, 2001)

\section{(2) Complementary Antonymy}

Complementary antonymy refers to the semantic field formed by words with complementary meanings. For example, dead-alive, If he is not dead, he is alive. / If he is not alive, he is dead. There are other such complementary antonymies, such as male-female, single-married, present-absent, etc. Differentiated with polar antonymy, normally speaking, there are no words which could be inserted between the pair of complementary words.

(3) Transpositional Antonymy

Transpositional antonymy is formed by a pair of words with both opposed and dependant meanings, the two words combined into an opposed unit which exists based on each other's existence. For example, buy-sell, lend-borrow, husband-wife, employer-employee, etc. Hence, If A lends \$100 to B, then we can say B borrows \$100 from A; If A is B' wife, then B is A's husband.

\section{Synonymy}

Synonymy refers to the semantic fields which are formed by the words with relatively same meanings so that they could be inter-changed in some degree yet irreplaceable in most cases. Synonymy could be further classified into absolute synonymy and relative synonymy. Absolute synonymy is relatively rare, and it mainly indicates the word groups with absolute same meaning and could be replaced with each other in any contexts. For instance, mother tongue and native language, malnutrition and undernourishment, breathed consonant and voiceless consonant, etc. While compared to absolute synonymy, relative synonymies are more affluent. It refers to the synonymies which are formed by words with relatively same meaning yet are different in degrees, emotions, styles and collocations. Thus, in reference to what Zhang yunfei (2004) made in her book An Introduction to Modern English Lexicology, relative synoynym could be further classified into such categories as following.

(1) Synonymy with Different Degrees

Words that constitute this type of semantic fields have same extended meanings yet different in degrees. For example, anger, rage and fury are all about the emotional excitement induced by intense displeasure, while anger is used normally without definite degree of intensity; rage focuses on a loss of self-control; and fury emphasizes a rage is so violent that it may approach madness.

(2) Synonymy with Different Emotions

This type of synonymy is mainly formed by words with basically same meaning yet with different emotions. For example, philanthropist and do-gooder can both means charity and sympathy, while philanthropist usually means charitarians who aid people in sufferings and donate for the poor, yet do-gooders refer to those unrealistic humanists who would dream of doing good recklessly and vainly. Another example, statesman and politician both means people who take part in social politics, while statesman refers to those who could do well in controlling and governing the sates yet politician mainly means people who play political tricks for their own interests. In both of the examples, the formers are all commendatory; the latters derogatory.

(3) Synonymy with Different Styles

This type of semantic field mainly consists of the words with relatively same meaning yet different styles. American linguist Martin Joos (1962) listed five different styles of words in his book Five Clocks as frozen, formal, consultative, casual and intimate. According to these five styles, we can label the word horse with five different tags, charger is frozen, steed is formal, horse is consultative, nag is casual, and plug is intimate.

(4) Synonymy with Different Collocations 
This type of synonymous semantic field is formed by words with relatively same meaning yet different collocations. For example, the words that could indicate the meaning of accuse are charge, rebuke, reproach and accuse itself. While the prepositions that collocate with them are different. That is to say, accuse collocates with of, charge collocates with with, rebuke collocates with for, and reproach with for or with.

\section{The ApPlication of Semantic Field Theory to Vocabulary LEARning}

\section{A. The Application of Hyponymy}

Semantic fields are obviously hierarchical. They could be observed from the top to the bottom. The higher level of a semantic field is more general, the lower level is more specific. For example:

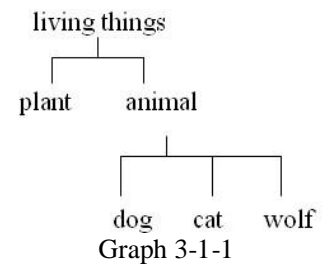

The hierarchy of semantic field is based on the systematization of objective substances. Meanwhile, it also reflects the consequences of the cognition of human being towards nature. Obviously, hierarchy is mostly seen in hyponymy. With this characteristic, we could learn new words more easily by studying the hyponyms or superordinates of the word we learn from a certain semantic field.

Another feature of semantic field is transitivity. That is to say, if $A$ belongs to $B$, and $B$ belongs to $C$, we can say that $A$ belongs to $C$. Transitivity is mainly reflected from two aspects, one of which could be found in the superordinate-hyponym semantic field that if $B$ is one type of $A$, and $C$ is one type of $B$, then we can say that $C$ is one type of $A$. For example, animal is one of the living things, and dog is one of the animals, then we can say dog is one of living things. On the other hand, transitivity could be found in part/whole relationship that if $B$ is part of $A, C$ is part of $B$, and then we can say $C$ is part of $A$. For example, season is a part of a year, month is a part of season, that makes month a part of a year.

Semantic fields usually tend to be complicated, that is why they have crisscross as one of the features. It mainly reflects from two aspects. On the one hand, a word with just one meaning could form different semantic fields with different words. For example, parent could form a hyponymy with father or mother; it could also form an antonymy with child. On the other hand, a word with different meanings could form various semantic fields with various words. For example, fast could form an antonymy with slow; meanwhile, it could also form a synonymy with words like quick, rapid and speedy, etc. and form another synonymy with firm and fixed. Another example, the word cow could not only refer the generic term of the semantic field of cattle in the higher level, but also could refer to simply the female cattle at a lower level in the same semantic field.

From the characteristics listed above, we can say that we could use the superordinates to sum up the words that belong to the same hyponymy and describe all other words that belong to the same hyponymy with the superordinates to make the context concise and clear as it is necessary. This approach also applies when it is unnecessary to list all the hyponyms. For example, we can use stationery to cover pens, pencils, rulers and paper, etc. when it is not necessary to list all these words. We can also use furniture to include chairs, tables, desks and beds, etc.

When learning about Jupiter, one of the planets of the solar system, English learners could expand the learning list to other names of planets of the solar system, such as Mars, Mercury, Saturn, Venus and so on. In an interestingly similar way, when learn about Mars, we might also learn that it is also the name of god of the war in ancient Roman legendaries, and Jupiter is the name of the king of all gods in the same system. This way, English learners could remember words much easily and systematically.

\section{B. The Application of Antonymy}

Generally, antonyms are used to indicate contrast and highlight the features of objects. Successful writers even use antonymy in the titles of their writings. Jane Austen is particularly fond of adopting this technique in her novels, such as Sense and Sensability, Pride and Prejudice. Antonymy helps form a sharp contrast and makes the works fascinating so that readers could continue reading with great interest.

Meanwhile, antonymy is often used to explain lexical meanings. Some words are difficult to explain directly, however, it will be easier to paraphrase using its antonym. For example, muscular, disgraceful, mediocre, and impoverishment might be unfamiliar to most students and make it difficult to explain in English, yet we can use not weak, not honored, not outstanding, and enrichment to directly explain them. Also, antonymy could be used to enlarge the vocabulary through covering pairs of semantically opposed antonyms. For example, when learning about employer, trainer and sender, we can simultaneously memorize employee, trainee and recipient.

G. Leech (1981) recognizes seven types of meaning in his Semantics. One is collocative meaning referring to what is communicated through association with words which tend to occur in the environment of another word. Linguistic 
context is just the collocation of one word with another. Some words are of polesemy. In different contexts, they have different meanings. Therefore, these words have different collocations respectively. Meanwhile, each word has different antonyms when its collocation changes.

Take fresh for example. The basic meaning of fresh is "newly coming out". Its antonym is "old". That is, the antonym of "fresh problem" is "old problem". When the meaning of fresh turns to "newly-made", its antonym turns to "state", that is, "no longer fresh". The antonym of "fresh bread" is "state bread". "Fresh fruit" means "newly gathered fruit", the fruit that are "not preserved" and "not dried". In this situation, the antonym of fresh becomes "preserved" or "dried". When fresh collocates with "flower", it means "newly gathered". The antonym of "fresh" is "faded" or "withered" accordingly. And when fresh collocates with "air", it refers to "clean and refreshing". Accordingly, its antonym is "foul" or "polluted". It is self-evident how vocabulary is easily enlarged from a single word "fresh" to about ten words or phrases by way of putting words in their semantic field of antonymy.

\section{The Application of Synonymy}

Seeing from a historical perspective, any language is always changing as time passes. Among all the changes of a language, the change of vocabulary is spectacular. It does not only indicate the vanishing of old words and the emergence of new words, but also could represent the change of the lexical meaning. Thus this change must have caused the change of semantic field. For example, the word nice used to mean stupid, which made it possible to form a synonymy with foolish and moronic; while in modern English, nice changes its meaning into good and fine, which makes them a new synonymy. Another example, the word bird used to mean squab, while nowadays becomes a generic term of all birds. Due to the change of meaning, a word would transfer from one semantic field to another.

Vagueness is another characteristic of semantic field. It indicates that due to the uncertain cognition of some objects or concepts from the external world of modern people and makes them vague. For example, it is still vague to decide whether to put the word olive in the semantic field of fruits or vegetables. Also, it is uncertain whether to put the word sled into the semantic filed of transportation or sport equipment.

In general, the application of synonymy could be used in describing the unfamiliar words. We could use the relativity and synonymy semantic field to enlarge vocabulary like what hyponymy and antonymy do; besides, vagueness of semantic fields could also help English learners distinguish different synonymy. As for synonymy itself, during learning process, there are a lot of words that are difficult to explain directly in English, in that case, we can use the synonyms to paraphrase them and acquire an easier access to learning their meanings. For example, we could use native language to directly paraphrase mother tongue.

During the vocabulary learning process, we can list up and compare the words that have similar semantic meaning and slight difference in general usage. For example, when learning about the word raise, we can list rise and arise as well. The three words share basic same meaning yet they are slightly different in grammar usage. Through this way, English learners could memorize both the spellings and specific usage of similar yet different words.

\section{CONCLUSION}

Vocabulary is one of the important elements in a language. English language is no exception. The efficiency of vocabulary learning greatly determines the success of language learning. English words are over a million in number and they constitute the core of language learning. The size of one's vocabulary directly affects the development of his/her linguistic competence. Therefore, the first task of vocabulary instruction is to enlarge learners' vocabulary. (Guo, 2010) Thus lots of vocabulary learning strategies have been recommended. However, the most systematic and scientific strategy should be the one based on semantic field theory.

This paper studies English vocabulary learning based on semantic field theory. In introduction, the paper presents the significance of the study and the theoretical review of semantic field. Words do not stand alone, rather, they form numerous dependently interrelated networks, either superordinate-hyponym, or antonymous, or synonymous, etc. Thus, the paper classifies the semantic field theory into three major categories, i.e., hyponymy, antonymy and synonymy, each of which are illustrated with abundant examples. Then the body of the paper is to apply each category of semantic relations to English vocabulary learning respectively with detailed elaboration. Meanwhile, the characteristics of each semantic field are summarized during the illustration, which include hierarchy, transitivity, crisscross, relativity, variability, vagueness, etc.

In conclusion, the paper only covers three major semantic relations, hyponymy, antonymy and synonymy. As for other semantic relations like polysemy, homonymy, taxonymy, partonymy, they do play vital roles in semantic field study as well as in vocabulary learning and teaching, and deserve researchers' further exploration.

\section{REFERENCES}

[1] Guo, Changhong. (2010). The Application of the Semantic Field Theory in College English Vocabulary Instruction. Chinese Journal of Applied Linguistics, 33, 50-62.

[2] Leech, Geoffrey. (1981). Semantics ( $2^{\text {nd }}$ edition). Harmondsworth: Penguin Books.

[3] Lewis, M. (1993). The Lexical Approach. London: Language Teaching Publications.

[4] Martin, J. (1962). The Five Clocks. Bloomington: Indiana University Research Center in Anthropology, Folklore and 
Linguistics.

[5] Mei, Jiaju, Zhu Yiming, Gao Yunqi \& Yin Hongxiang. (1987). Semantic Field and Semantic System. Foreign Languages, 49, $18-23$.

[6] Nation, P. (2001). Learning Vocabulary in Another Language. Cambridge: Cambridge University Press.

[7] Wang, Rongpei \& Wang, Zhijiang. (2008). Manual of English Lexicology. Shanghai: Shanghai Foreign Language Education Press.

[8] Wilkins, D. A. (1972). Linguistics in Language Teaching. London: Edward Arnold.

[9] Wu, Qianguang. (1988). Introduction to Semantics. Changsha: Hunan Education Press.

[10] Zhou, Weijie. (1997). A Research on English Semantic Field. Shandong Foreign Languages Journal, 68, 21-23.

[11] Zhou, Weijie. (2001). A New Research on English Semantic Field. Journal of Beijing International Studies University, 102, 30-35.

[12] Zhang, Yunfei. (2004). An Introduction to Modern English Lexicology. Beijing: Beijing Normal University Press.

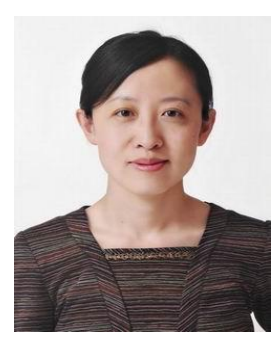

Chunming Gao was born in 1979 in Jilin Province, China. She received her M.A degree in Linguistics and Applied Linguistics in Foreign Languages from Changchun University of Science and Technology in 2006. She is currently a lecturer in the School of Foreign Languages, Changchun University of Science and Technology, Changchun, China. Her major research interests include applied linguistics and cross-cultural communication.

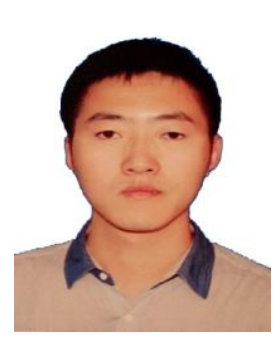

Bin Xu was born in 1990 in Jilin Province, China. He received his B.A. degree from Changchun University of Science and Technology in 2013. He is currently studying at Kanrin Japanese School in Japan as a language learner. 